\title{
Dirac and Klein-Gordon particles in one-dimensional periodic potentials
}

\author{
Michaël Barbier, ${ }^{1, *}$ F. M. Peeters, ${ }^{1, \dagger}$ P. Vasilopoulos, ${ }^{2, \ddagger}$ and J. Milton Pereira, Jr. ${ }^{1,3, \S}$ \\ ${ }^{1}$ Department of Physics, University of Antwerp, Groenenborgerlaan 171, B-2020 Antwerpen, Belgium \\ ${ }^{2}$ Department of Physics, Concordia University, 7141 Sherbrooke Ouest Montreal Quebec, Canada, H4B 1R6 \\ ${ }^{3}$ Departamento de Física, Universidade Federal do Ceará, Fortaleza, Ceará 60455-760, Brazil
}

(Received 25 October 2007; published 25 March 2008)

\begin{abstract}
We evaluate the dispersion relation for massless fermions, described by the Dirac equation, and for zero-spin bosons, described by the Klein-Gordon equation, moving in two dimensions and in the presence of a onedimensional periodic potential. For massless fermions, the dispersion relation shows a zero gap for carriers with zero momentum in the direction parallel to the barriers in agreement with the well-known "Klein paradox." Numerical results for the energy spectrum and the density of states are presented. Those for fermions are appropriate to graphene in which carriers behave relativistically with the "light speed" replaced by the Fermi velocity. In addition, we evaluate the transmission through a finite number of barriers for fermions and zero-spin bosons and relate it with that through a superlattice.
\end{abstract}

DOI: 10.1103/PhysRevB.77.115446

PACS number(s): 71.10.Pm, 73.21.-b, 81.05.Uw

\section{INTRODUCTION}

The recent realization of stable-single layer and bilayer carbon crystals (graphene) has aroused considerable interest in the study of their electronic properties. ${ }^{1,2}$ These materials have unusual properties that may allow the development of carbon-based nanoelectronic devices. The behavior of charge carriers in wide single-layer graphene sheets is that of chiral, "relativistic" massless particles with a "light speed" equal to the Fermi velocity of the crystal $\left(v_{F} \approx c / 300\right)$ and a gapless linear dispersion close to the $K$ and $K^{\prime}$ points. ${ }^{1,3,4}$ One consequence of that is that single-layer graphene displays an unusual quantum Hall effect, in which the quantum Hall plateaus occur ${ }^{4}$ at half-integer multiples of $4 e^{2} / h$. The absence of a gap and the chiral nature of the electronic states, in both single-layer and bilayer graphene, is at the root of phenomena such as the Klein tunneling ${ }^{5-8}$ which is the perfect transmission of carriers, upon normal incidence, through a potential barrier. The study of this effect is relevant to the development of future graphene-based devices. ${ }^{9}$

From the standpoint of basic research, due to its lower light speed, graphene can also fulfill the role of a testbed for the investigation of relativistic quantum effects, such as Zitterbewegung ${ }^{10}$ and pair creation. ${ }^{11}$ Moreover, the role of chirality in the electronic properties of massless fermions can be assessed or further appreciated by contrasting the behavior of chiral, massless fermions with that of nonchiral, massless zero spin bosons.

In this work, we study the dispersion relation for twodimensional fermions described by the Dirac equation in the presence of a one-dimensional (1D) periodic potential. This can be realized in a periodically gated graphene layer. In doing so, we extend the treatment of Ref. 12 which considered motion only along one direction. For a clearer understanding, we contrast these results with those for bosons of zero spin that are described by the Klein-Gordon equation. ${ }^{13}$ Here, too, we include the motion parallel to the barriers, which, to our knowledge, has not yet been reported. In addition, we briefly present results, in Sec. II, for the transmission through a single barrier. In Sec. III, we present the dis- persion relation and transmission for either superlattice case and relate the transmission to that through a single barrier. A summary and concluding remarks follow in Sec. VI.

\section{TUNNELING THROUGH A SINGLE BARRIER}

\section{A. Bosons}

We first calculate the transmission of zero-spin bosons through a single barrier of height $V$ and width $W$, shown in Fig. 1, using the Klein-Gordon equation in two dimensions,

$$
\nabla^{2} \psi(x, y)=-\frac{1}{\hbar^{2} c^{2}}\left\{[E-V(x)]^{2}-m^{2} c^{4}\right\} \psi(x, y) .
$$

Since the Hamiltonian $H$ commutes with $p_{y}$, we look for solutions in the form $\psi(x, y)=\psi(x) e^{i k_{y} y}$. With this substitution, the resulting equation for $\psi(x)$ is solved by $\psi(x)$ $=e^{ \pm i k_{x} x}$ outside the barrier with $k_{x}=E^{\prime} / \hbar c$ and by $\psi(x)$ $=e^{ \pm i K x}$ inside it with

$$
K=\frac{1}{\hbar c}\left[E^{\prime 2}-2 V\left(E^{\prime 2}+\hbar^{2} k_{y}^{2} c^{2}+m^{2} c^{4}\right)^{1 / 2}+V^{2}\right]^{1 / 2},
$$

and with $E^{\prime}$ given by

$$
E^{\prime 2}=E^{2}-\hbar^{2} k_{y}^{2} c^{2}-m^{2} c^{4}=\hbar^{2} k_{x}^{2} c^{2} .
$$

Then, following the standard procedure of matching the solutions and their derivatives at the interfaces of regions I and II, and II and III, shown in Fig. 1, leads to the transmission

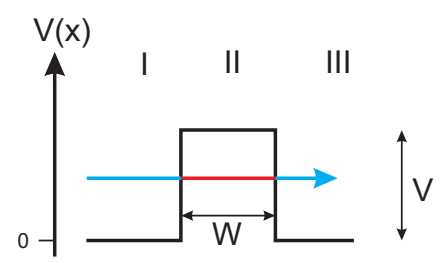

FIG. 1. (Color online) 1D Potential barrier $V(x)$ of height $V$ and width $W$. 


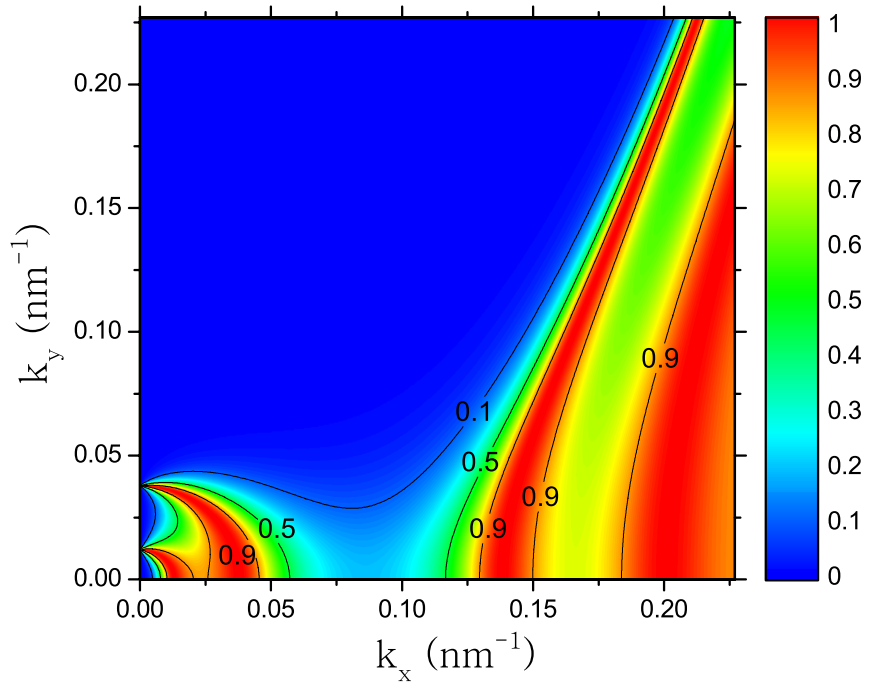

FIG. 2. (Color online) Contour plot of the transmission $\mathcal{T}$ of relativistic bosons through a barrier as a function of $k_{x}$ and $k_{y}$ for $V=50 \mathrm{meV}, c=10^{6} \mathrm{~m} / \mathrm{s}, W=50 \mathrm{~nm}$, and $m=0$.

$$
\mathcal{T}\left(k_{x}, k_{y}\right)=\left[1+\left(\frac{k_{x}^{2}-K^{2}}{2 k_{x} K}\right)^{2} \sin ^{2}(K W)\right]^{-1} .
$$

If $K$ becomes imaginary, then $K$ is replaced by $i|K|$ and $\sin (K W)$ by $i \sinh (K W)$ in this equation. Notice that in contrast to the nonrelativistic case, $K$ depends on $k_{y}$. The result for the nonrelativistic case is obtained by inserting $k_{y}=0$, $k_{x}=\left[2 m E / \hbar^{2}\right]^{1 / 2}$, and $K=\left[2 m(E-V) / \hbar^{2}\right]^{1 / 2}$ in Eq. (4).

In Fig. 2, the transmission through a single potential barrier is plotted for zero-spin bosons with the same velocity $\left(c=10^{6} \mathrm{~m} / \mathrm{s}\right)$ and parameters for the potential barrier as for the massless fermions in graphene. In doing so, we will be able to better compare the transmission for bosons with that for fermions. As shown in Fig. 4, in which we plot slices of this plot at constant $k_{y}$, the transmission is perfect for certain values of $k_{x}$, although the boson has an energy that is much lower than the height of the potential barrier $V$ (if $k_{y}=0$, then $E=V=50 \mathrm{meV}$ for $k_{x}=0.076 \mathrm{~nm}^{-1}$ ). Notice that these transmission resonances below the barrier height do not occur in the nonrelativistic case. Moreover, the transmission depends on the wave vector component parallel to the barrier, i.e., on $k_{y}$ since $K$ does [cf. Eq. (2)], whereas it does not in the nonrelativistic case.

Since $K$ is always real for $m=0$, the sine term in Eq. (4) only introduces a modulation of the transmission. The minima in the transmission arise from the $\left(k_{x}^{2}-K^{2}\right) / 2 k_{x} K$ term. This is because at the vicinity of $E=V$, the solutions inside the barrier approach those of a free particle with zero energy and thus with $k=0$, i.e., infinite wavelength. Together with the continuity of $\Psi$ and of its derivative, this acts to reduce the amplitude of the transmitted wave. For the nonrelativistic case and $E<V, K$ becomes imaginary and the sine term becomes a hyperbolic sine, which can become quite large and reduce significantly the total transmission.

\section{B. Fermions}

In two dimensions, spin- $1 / 2$ fermions are described by the two-dimensional Dirac equation

$$
\left[c(\vec{\sigma} \cdot \hat{p})+m c^{2} \sigma_{z}\right] \Psi=(E-V) \Psi,
$$

where $\vec{\sigma}=\left(\sigma_{x}, \sigma_{y}\right)$ and $\sigma_{z}$ are the Pauli matrices. Instead of imposing a certain form of solution, without giving any details, to Eq. (5), ${ }^{6}$ we look for solutions in the form $\psi(x, y)$ $=\psi(x) e^{i k_{y} y}$, where the wave function $\psi(x)$ is a twocomponent spinor $\psi(x)=\left[\psi_{u}(x), \psi_{l}(x)\right]^{T}$. Substituting this form of solution and setting $E^{\prime}=E-V(x)$ gives

$$
\begin{aligned}
& \partial \psi_{l}(x) / \partial x+k_{y} \psi_{l}(x)=\frac{i}{\hbar c}\left(E^{\prime}-m c^{2}\right) \psi_{u}(x), \\
& \partial \psi_{u}(x) / \partial x-k_{y} \psi_{u}(x)=\frac{i}{\hbar c}\left(E^{\prime}+m c^{2}\right) \psi_{l}(x) .
\end{aligned}
$$

These coupled equations admit solutions of the form

$$
\psi(x)=\left(\begin{array}{l}
u_{u} \\
u_{l}
\end{array}\right) e^{i k_{x} x}
$$

where $k_{x}=(1 / \hbar c)\left[E^{2}-\hbar^{2} k_{v}^{2} c^{2}-m^{2} c^{4}\right]^{1 / 2}$ must be replaced by $K=(1 / \hbar c)\left[(E-V)^{2}-\hbar^{2} k_{y}^{2} c^{2}-m^{2} c^{4}\right]^{1 / 2}$ inside the barrier. Then, substituting this form in Eq. (5) gives

$$
\left(\begin{array}{cc}
E^{\prime}-m c^{2} & -\hbar c k_{x,-} \\
-\hbar c k_{x,+} & E^{\prime}+m c^{2}
\end{array}\right)\left(\begin{array}{l}
u_{u} \\
u_{l}
\end{array}\right)=0
$$

where $k_{x, \pm}=k_{x} \pm i k_{y}$. A nontrivial solution demands a zero determinant and this leads to

$$
E^{\prime 2}=\hbar^{2} c^{2} k_{x}^{2}+\hbar^{2} c^{2} k_{y}^{2}+m^{2} c^{4}
$$

and to

$$
\begin{aligned}
& u_{l}=\frac{\hbar c k_{x,+}}{E^{\prime}+m c^{2}} u_{u}, \quad E^{\prime}>0, \\
& u_{u}=\frac{-\hbar c k_{x,-}}{\left|E^{\prime}\right|+m c^{2}} u_{l}, \quad E^{\prime}<0 .
\end{aligned}
$$

As detailed in the Appendix, the general solution is a linear combination of two independent solutions $\phi_{a}(x)$ and $\phi_{b}(x)$ of this form. In contrast to the boson case of Sec. II A, the continuity of the derivative of the spinor components for Dirac fermions is not necessary. Thus, matching only the solutions at the interfaces between regions I and II and II and III gives, for $E>V$, the transmission as

$$
\mathcal{T}\left(k_{x}, k_{y}\right)=\left[1+\left(\frac{F}{4 k_{x}^{2} K^{2}}-1\right) \sin ^{2}(K W)\right]^{-1},
$$

where

$$
F=\left[k_{x}^{2} \frac{D}{d}+K^{2} \frac{d}{D}+k_{y}^{2}\left(\frac{d}{D}+\frac{D}{d}-2\right)\right]^{2},
$$

with $d=\left(|E|+m c^{2}\right) / \hbar c$ and $D=\left(|E-V|+m c^{2}\right) / \hbar c$. Similar expressions are obtained for $0<E<V$ and $E<0$ (cf. the Appendix).

For $|E-V|^{2}<\hbar^{2} k_{y}^{2} c^{2}+m^{2} c^{4}, K$ is imaginary and the transmission is given by Eq. (11) with $K$ replaced by $i K^{\prime} \equiv i|K|$ and $\sin (K W)$ by $i \sinh (K W)$. A contour plot of the transmission is given in Fig. 3. As can be seen, for normal incidence, 


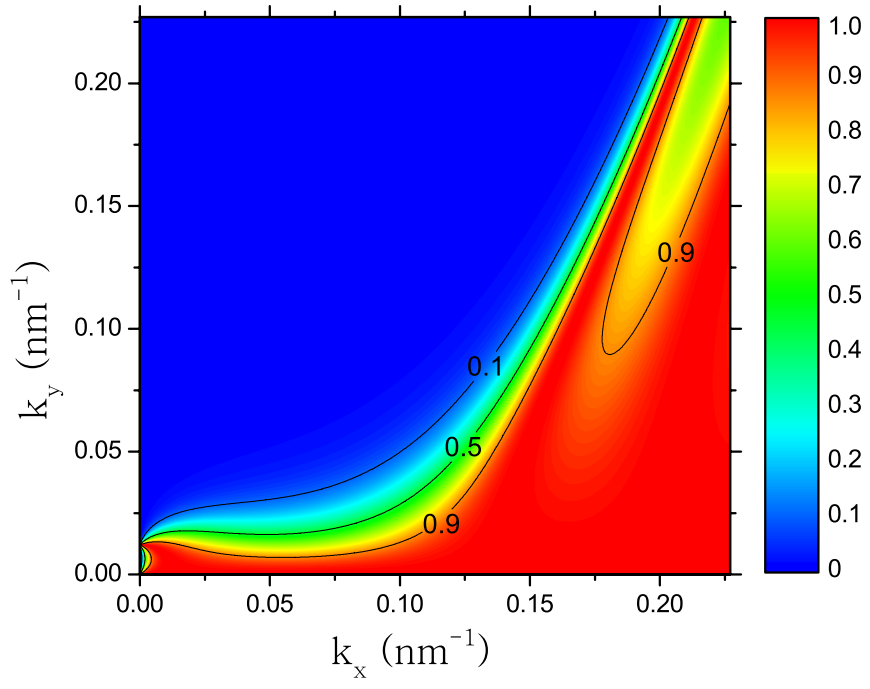

FIG. 3. (Color online) Contour plot of transmission $\mathcal{T}$ of massless relativistic electrons through a barrier with $m=0, V=50 \mathrm{meV}$, $c=10^{6} \mathrm{~m} / \mathrm{s}$, and $W=50 \mathrm{~nm}$.

i.e., for $k_{y}=0$, the transmission is equal to 1 . Contrasting Fig. 3 with Fig. 2 for bosons, we see a significant gap in Fig. 2 for normal incidence that is not present in Fig. 3. Another way to contrast the results of the two cases is shown in Fig. 4 where slices of the transmission across the planes $k_{y}=0$ and $k_{y}$ $=0.05 \mathrm{~nm}^{-1}$ are shown for bosons (green curves) and electrons (red and magenta curves). The perfect transmission $\left[\mathcal{T}\left(k_{x}, k_{y}\right)=1\right]$ for normal incidence results from the chirality of the carriers which prevents them from being reflected by a barrier (see also Ref. 7). Because massless bosons are not chiral, they are not subject to such a constraint.

\section{SUPERLATTICES}

We now consider 1D superlattices (SLs) that are periodic structures consisting of $N$ identical units of length $L$. Each

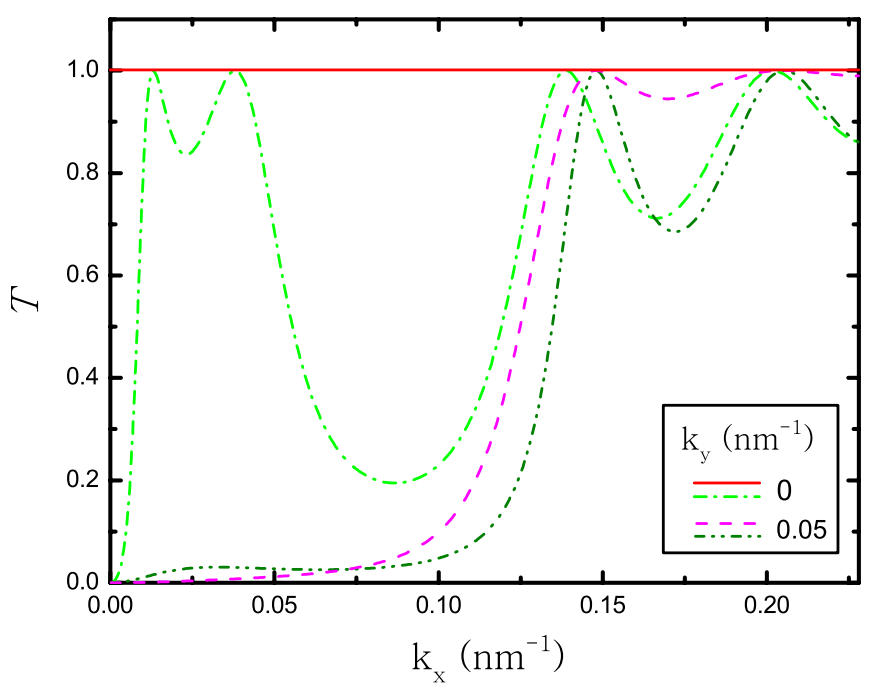

FIG. 4. (Color online) Plot of slices of the transmission coefficient for bosons (green dot- (dot)-dashed curves) and electrons (solid red and dashed magenta curves) taken at constant $k_{y}=0$ and $k_{y}=0.05 \mathrm{~nm}^{-1}$ from Figs. 2 and 3, respectively. unit consists of a rectangular barrier and a rectangular well whose widths are denoted, respectively, by $a$ and $b$. We first consider fermions described by the Dirac equation [Eq. (5)]. For motion of electrons along only one direction $(x)$, such a calculation was done by McKellar et al. ${ }^{12}$ We generalize this earlier work to two dimensions.

We start with Eqs. (5)-(7) taking $c \approx 10^{6} \mathrm{~m} / \mathrm{s}$ for our numerical results, a value that is appropriate for graphene. Equation (7) can be written as

$$
\psi(x)=\left(\begin{array}{l}
\psi_{u}(x) \\
\psi_{l}(x)
\end{array}\right) .
$$

Using Eq. (5), we have two independent solutions $\phi_{a}(x)$ and $\phi_{b}(x)$ in each unit cell. The solution in the $n$th unit cell $n$, written as $\psi_{n}(x)=\psi[x+(n-1) l]$, is a linear combination of these two,

$$
\psi_{n}(x)=a_{n}\left(\begin{array}{c}
\phi_{a, u}(x) \\
\phi_{a, l}(x)
\end{array}\right)+b_{n}\left(\begin{array}{c}
\phi_{b, u}(x) \\
\phi_{b, l}(x)
\end{array}\right), \quad x \in[0, l] .
$$

It is convenient to define the matrix

$$
\boldsymbol{\Omega}(x)=\left(\begin{array}{cc}
\phi_{a, u}(x) & \phi_{b, u}(x) \\
\phi_{a, l}(x) & \phi_{b, l}(x)
\end{array}\right) .
$$

Then, the continuity of the wave function leads to the following relation between the coefficients of the unit cells:

$$
\begin{gathered}
\boldsymbol{\Omega}(0)\left(\begin{array}{l}
a_{n+1} \\
b_{n+1}
\end{array}\right)=\boldsymbol{\Omega}(l)\left(\begin{array}{l}
a_{n} \\
b_{n}
\end{array}\right), \\
\Rightarrow\left(\begin{array}{l}
a_{n+1} \\
b_{n+1}
\end{array}\right)=\mathbf{T}\left(\begin{array}{l}
a_{n} \\
b_{n}
\end{array}\right),
\end{gathered}
$$

and the transfer matrix $\mathbf{T}$ is given by $\mathbf{T}=\boldsymbol{\Omega}(0)^{-1} \boldsymbol{\Omega}(l)$.

It can be proven ${ }^{12}$ that $\operatorname{det}[\mathbf{T}]=1$. Assuming periodic boundary conditions, we can write

$$
\begin{gathered}
\psi(x+L)=\psi(x), \\
\Rightarrow\left(\begin{array}{l}
a_{N+n} \\
b_{N+n}
\end{array}\right)=\left(\begin{array}{l}
a_{n} \\
b_{n}
\end{array}\right),
\end{gathered}
$$

and obtain

$$
\begin{gathered}
\left(\begin{array}{l}
a_{N+n} \\
b_{N+n}
\end{array}\right)=\mathbf{T}^{N}\left(\begin{array}{l}
a_{n} \\
b_{n}
\end{array}\right), \\
\Rightarrow \mathbf{T}^{N}=1 .
\end{gathered}
$$

It follows that the eigenvalues of $\mathbf{T}$ are $e^{2 \pi i n / N}$, with $n$ as an integer, and its determinant is equal to 1 . The dispersion relation is then given by the trace of $\mathbf{T}$ as

$$
2 \cos \left(k_{x} l\right)=\operatorname{Tr}(\mathbf{T}),
$$

where $k_{x}=2 \pi n / L$. Notice that this $k_{x}$, or Bloch wave vector, expresses the periodicity of the structure and should not be confused with the $k_{x}$ of Sec. II.

\section{A. One-dimensional superlattices in graphene}

The dispersion relation is given by Eq. (22), and the transfer matrix $\mathbf{T}$ by 


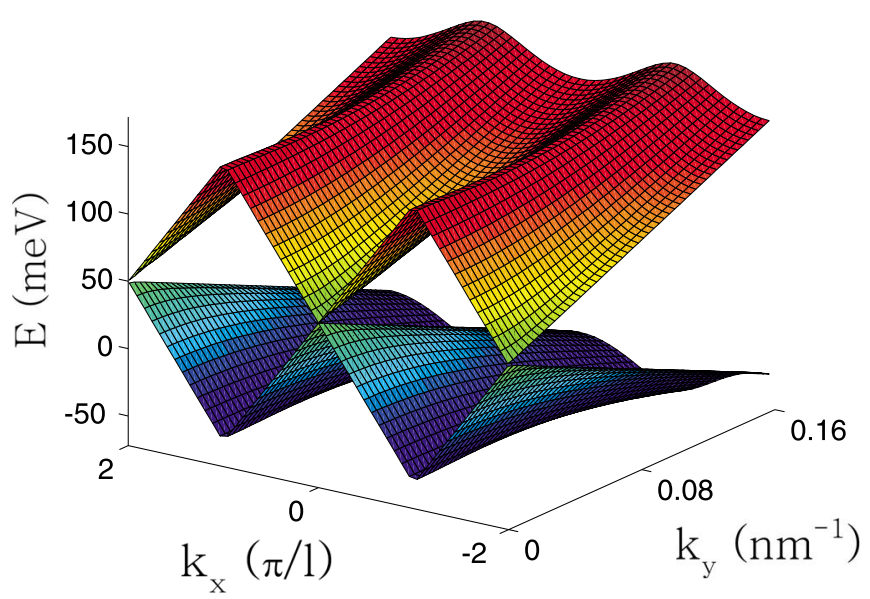

FIG. 5. (Color online) Dispersion relation in a $1 \mathrm{D}$ graphene SL. Only the first two bands are shown for $a=b=10 \mathrm{~nm}, V=100 \mathrm{meV}$, and $m=0$.

$$
\mathbf{T}=\boldsymbol{\Omega}_{\kappa}(l) \boldsymbol{\Omega}_{\kappa}(a)^{-1} \boldsymbol{\Omega}_{K}(a) \boldsymbol{\Omega}_{K}(0)^{-1},
$$

where $\boldsymbol{\Omega}_{K}$ and $\boldsymbol{\Omega}_{\kappa}$ are the matrices in the barriers and wells, respectively. With the help of the Appendix, we can specify the matrices $\boldsymbol{\Omega}_{K}$ and $\boldsymbol{\Omega}_{\kappa}$. For $E>V$, the dispersion relation becomes $\left(\kappa=E^{\prime} / \hbar c\right)$

$$
2 \cos \left(k_{x} l\right)=2 \cos (K a) \cos (\kappa b)-G \sin (K a) \sin (\kappa b),
$$

where

$$
G=\left(\frac{\kappa^{2}+k_{y}^{2}}{d^{2}}+\frac{K^{2}+k_{y}^{2}}{D^{2}}\right) \frac{d D}{\kappa K}-\frac{2 k_{y}^{2}}{\kappa K} .
$$

By setting $k_{y}=0$ in Eqs. (24) and (25), we recover the result of Ref. 12 for the 1D motion of Dirac particles. For $(E$ $-V)^{2}<m^{2} c^{4}+\hbar^{2} k_{y}^{2} c^{2}$ and $E-V>0$, we have an imaginary $K$ and simply make the substitution $K \rightarrow i|K|$ in Eq. (24). For $E<0$, the result for $G$ is the same, whereas for $V>E>0$, it becomes

$$
G=-\frac{d D}{K \kappa}-\frac{\left(K^{2}+k_{y}^{2}\right)\left(\kappa^{2}+k_{y}^{2}\right)}{K \kappa d D}-\frac{2 k_{y}^{2}}{\kappa K} .
$$

The first two minibands of the dispersion relation for a 1D graphene-based SL are plotted in Fig. 5. Notice that the form of the dispersion relation in the $y$ direction, in contrast to the nonrelativistic case, depends on the $x$ component $k_{x}$ of the wave vector and is not the energy of a free particle. As a result, we cannot split the energy of the minibands in the manner $E_{n}=E_{n}(x)+c \hbar k_{y}$. If $k_{y}$ is sufficiently large, this dependence goes away and we have again a linear relation between the energy and $k_{y}$, independent of the $k_{x}$ component. Notice also that for $k_{y}=0$ (and $m=0$ ), the linear dispersion relation $E\left(k_{x}, 0\right)=v_{F} \hbar k_{x}$ is unaltered by the periodic potential $V$; it is only shifted by $V / 2$. This is a consequence of the Klein paradox.

In Fig. 6, we show three slices of the dispersion relation
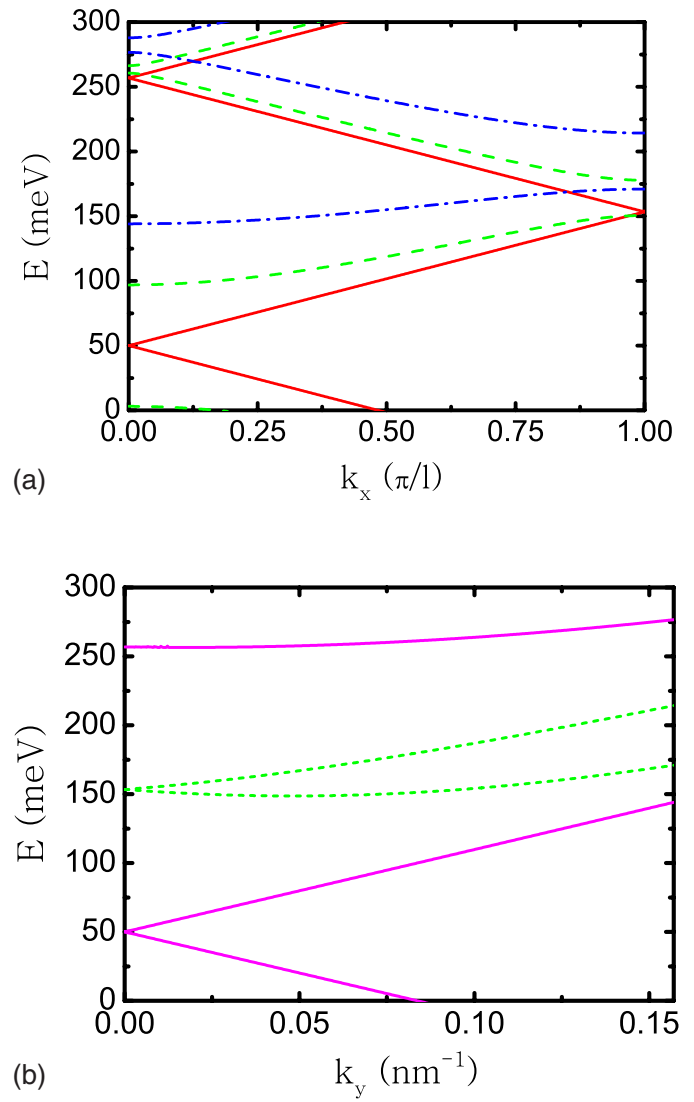

FIG. 6. (Color online) Slices of the dispersion relation shown in Fig. 5. (a) is for constant $k_{y}=0,0.066,0.132 \mathrm{~nm}^{-1}$ for the solid red, dashed green, and dash-dotted blue curves, respectively, and (b) for constant $k_{x}=0, \pi / l$ for the solid magenta and dashed green curves, respectively.

of Fig. 5, taken at constant $k_{y}=0,0.066,0.132 \mathrm{~nm}^{-1}$ (red solid, green dashed, and blue dash-dotted curves, respectively). We see that the $k_{y}$ component is also generating a band gap.

It is instructive to take the Kronig-Penney limit of the dispersion relations given above. For very thin and high barriers, such that $a \rightarrow 0$ and $V \rightarrow \infty$, but with their product $(S$ $=a V)$ kept constant we have

$$
\begin{gathered}
\kappa=\frac{\sqrt{E^{2}-m^{2} c^{4}}}{\hbar c}, \quad K=\frac{\sqrt{(E-V)^{2}-m^{2} c^{4}}}{\hbar c} \approx \frac{V}{\hbar c}, \\
\frac{\kappa}{d}=\frac{\sqrt{E^{2}-m^{2} c^{4}}}{|E|+m c^{2}}, \quad \frac{K}{D}=\frac{K \hbar c}{\left|E^{\prime}\right|+m c^{2}} \approx 1 .
\end{gathered}
$$

For $V \rightarrow \infty$, only the cases with positron waves in the barrier are allowed. Then, the dispersion relation becomes

(1) $|E|>m c^{2}$,

$$
2 \cos \left(k_{x} l\right)=2 \cos \left(\frac{S}{\hbar c}\right) \cos (\kappa l)+G_{+} \sin \left(\frac{S}{\hbar c}\right) \sin (\kappa l) .
$$

(2) $|E|<m c^{2}$, 


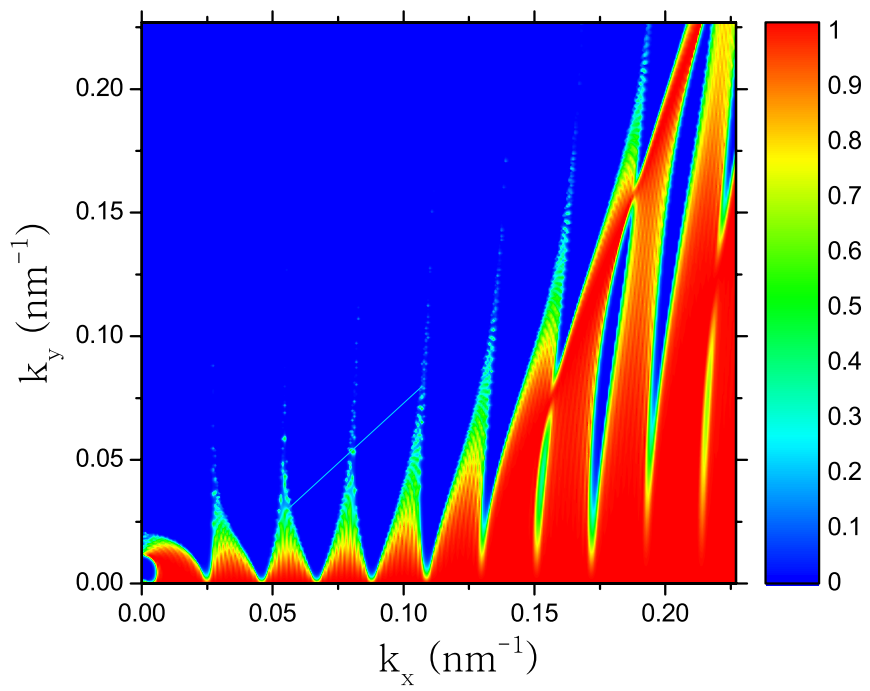

FIG. 7. (Color online) Contour plot of the transmission $\mathcal{T}$ of relativistic fermions in a structure consisting of ten units with $c$ $=10^{6} \mathrm{~m} / \mathrm{s}, V=50 \mathrm{meV}, a=50 \mathrm{~nm}, b=100 \mathrm{~nm}$, and $m=0$.

$$
2 \cos \left(k_{x} l\right)=2 \cos \left(\frac{S}{\hbar c}\right) \cosh (\kappa l)+G_{-} \sin \left(\frac{S}{\hbar c}\right) \sinh (\kappa l)
$$

where $G_{ \pm}=\kappa / d \pm d / \kappa+k_{y}^{2} / \kappa d$.

To make contact with Sec. II, we now consider the transmission through a multibarrier structure that is a good approximation to a SL. In Fig. 7, we show a contour plot of the transmission for a structure consisting of ten units. Upon contrasting Figs. 3 and 7, it is seen that the main effect of having many units is to slightly reduce the continuous range of $k_{x}$ for which a perfect transmission occurs by "inserting" small gaps between regions of perfect transmission. The resonances also show a very slight increase in the transmission. This behavior remains essentially unaltered if the number of units is increased and is similar to that reported previously in the context of resonant tunneling through a double barrier. $^{9}$

\section{B. Bosons}

The dispersion relation for a SL and relativistic bosons is obtained in a similar way and in full analogy with the nonrelativistic Schrödinger equation. We use the Klein-Gordon equation [Eq. (1)] and obtain the same dispersion relation as in the nonrelativistic case but with different values for $\kappa$ and $K$, namely,

$$
\begin{gathered}
\kappa=(1 / \hbar c)\left[E^{2}-\hbar^{2} k_{y}^{2} c^{2}-m^{2} c^{4}\right]^{1 / 2}, \\
K=(1 / \hbar c)\left[(E-V)^{2}-\hbar^{2} k_{y}^{2} c^{2}-m^{2} c^{4}\right]^{1 / 2} .
\end{gathered}
$$

The dispersion relation is

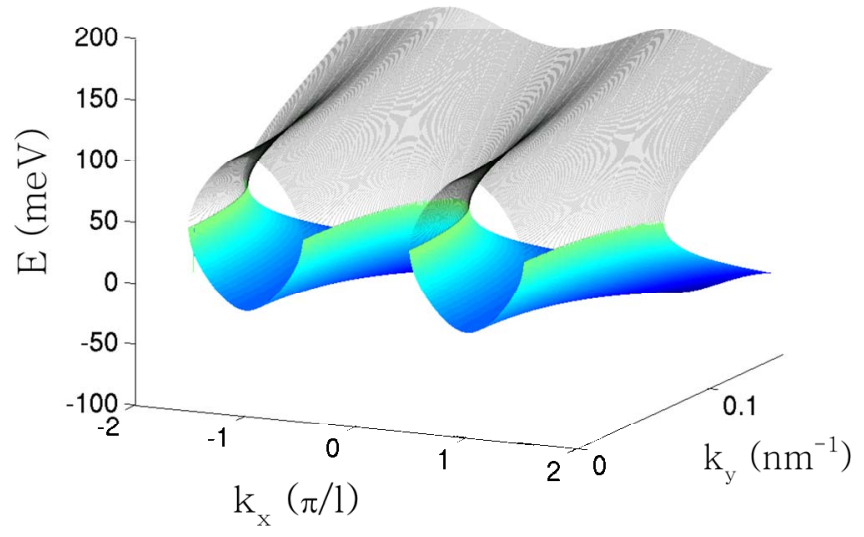

FIG. 8. (Color online) Dispersion relation for relativistic massless zero-spin bosons. Only the first two minibands are shown for $c=10^{6} \mathrm{~m} / \mathrm{s}, V=100 \mathrm{meV}, a=b=10 \mathrm{~nm}$, and $m=0$.

$$
2 \cos \left(k_{x} l\right)=2 \cos (K a) \cos (\kappa b)-\frac{\kappa^{2}+K^{2}}{\kappa K} \sin (K a) \sin (\kappa b) .
$$

If $K$ becomes imaginary, which happens for $(E-V)^{2}<m^{2} c^{4}$ $+\hbar^{2} k_{y}^{2} c^{2}$, we simply replace $K$ by $i|K|$ in Eq. (34).

In Fig. 8, we plot the first two minibands as a function of $k_{x}$ and $k_{y}$ for the parameters shown in the caption. The main difference with the nonrelativistic case is the appearance of a large gap along $k_{x}$ and part of $k_{y}$, centered around $k_{x}=0$. Notice the turning point of the band, which leads to an infinite group velocity $v_{k_{y}}$. Such an unusual result is not uncommon in connection with the Klein-Gordon equation. ${ }^{14}$ If we take the mass sufficiently different from zero, the turning point in the superlattice band structure disappears. Notice the similarity and differences with the relativistic fermion case depicted in Fig. 5. The situation is similar between Figs. 6 and 9, where the dispersion relation is shown vs $k_{x}$ in the left panels and vs $k_{y}$ in the right ones.

Again, we make contact with Sec. II by considering the transmission through a ten-unit structure described above for fermions. A contour plot of the transmission is shown in Fig. 10. Contrasting Figs. 2 and 10, we see that the main effect of having many units is to reduce the continuous range of $k_{x}$ for which a perfect transmission occurs, by inserting small gaps between regions of perfect transmission, and introduce resonances in the gap of Fig. 2 which occurs for $0.5 \leqslant k_{x}$ $\leqslant 0.014$. This was not the case for fermions for which the transmission was perfect in this range. Another way to contrast the results of the two superlattice cases considered above is shown in Fig. 11, where the transmission is plotted vs $k_{x}$ for ten units and constant $k_{y}$. Panel (a) is for normal incidence, that is, for $k_{y}=0$, the red horizontal line being for electrons, and the blue one for bosons. Panels (b) and (c) are for electrons and bosons, respectively, and in either case for $k_{y}=0.02 \mathrm{~nm}^{-1}$. We see again the perfect transmission for electrons in panel (a) and the quite different one for bosons while for an oblique incidence, the difference is less pronounced. Notice the quite "square-wave" character of the transmission, which becomes more pronounced upon in- 

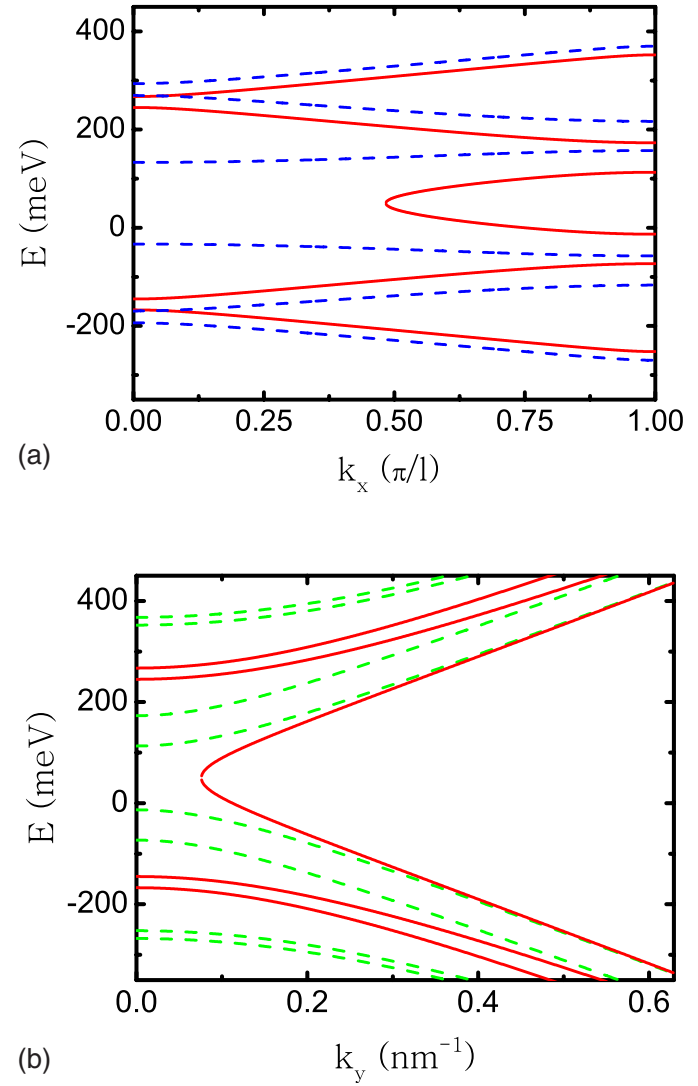

FIG. 9. (Color online) Slices of the dispersion relation for relativistic bosons extracted from Fig. 8. (a) is for constant $k_{y}=0$, $0.16 \mathrm{~nm}^{-1}$, for the solid red and dashed blue curves, respectively, and (b) for constant $k_{x}=0, \pi / \ell$, for the solid red and dashed green curves, respectively.

creasing the number of units. Notice also the similarity between Figs. 4 and 11. Again, the differences between electrons and bosons are due to the chirality of the former.

\section{Density of states}

An additional way to contrast electrons with bosons is to evaluate the density of states (DOS) $D(E)$. In the reducedzone scheme, it is given by

$$
D(E)=\frac{4 A}{\pi^{2}} \sum_{n} \int_{0}^{\pi / l} d k_{x} \int_{0}^{\infty} d k_{y} \delta\left[E-E_{n}\left(k_{x}, k_{y}\right)\right],
$$

where $A$ is the surface area. The integral is evaluated numerically by converting it to a sum in the manner

$$
\int_{0}^{\pi / l} d k_{x} \int_{0}^{\infty} d k_{y} \approx\left(\frac{\pi}{N_{x} l}\right)\left(\frac{C}{N_{y}}\right) \sum_{k_{x}=0}^{\pi / l} \sum_{k_{y}=0}^{C},
$$

where the $k_{x}$ and $k_{y}$ indices take the values

$$
k_{x}=\frac{n_{x}}{N_{x}} \frac{\pi}{l}, \quad k_{y}=\frac{n_{y}}{N_{y}} C, \quad n_{x}\left(n_{y}\right)=1 \cdots N_{x}\left(N_{y}\right) .
$$

The cutoff $C$ in the $k_{y}$ direction is chosen sufficiently large so that there is negligible change in the DOS by not considering

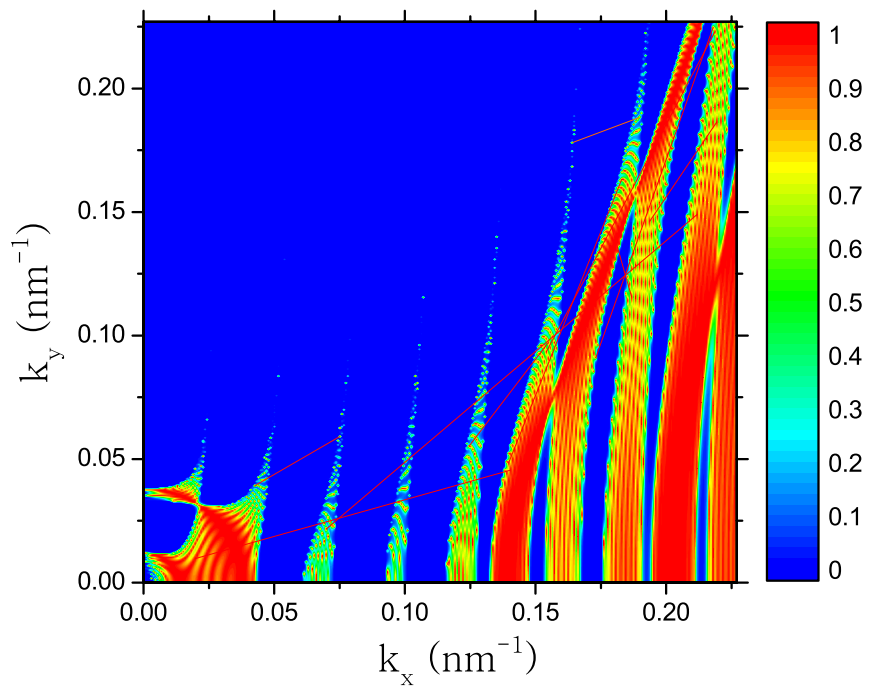

FIG. 10. (Color online) Contour plot of the transmission of relativistic bosons in a structure consisting of ten units with $c$ $=10^{6} \mathrm{~m} / \mathrm{s}, V=50 \mathrm{meV}, a=50 \mathrm{~nm}, b=100 \mathrm{~nm}$, and $m=0$.

contributions from $k_{y}>C$; for the plots shown, we took $C$ $=10 \pi / l$. In addition, we replace the $\delta$ function in Eq. (33) by a Gaussian, of width $\sigma=3 \mathrm{meV}$,

$$
\delta\left[E-E_{n}\left(k_{x}, k_{y}\right)\right] \approx \frac{1}{\sigma \sqrt{2 \pi}} e^{-\left[E-E_{n}\left(k_{x}, k_{y}\right)\right]^{2} / 2 \sigma^{2}},
$$

and chose $\sigma$ small but sufficiently large to compensate for the discretization of $k_{x}$ and $k_{y}$. For the DOS of zero-spin bosons, we divide the result by 4 since we do not have the fourfold valley-and-spin degeneracy. We also evaluate $D(E)$ for a nonrelativistic superlattice in the tight-binding model

$$
E_{n}\left(k_{x}, k_{y}\right)=E_{n}+\hbar^{2} k_{y}^{2} / 2 m-2 t_{n} \cos \left(k_{x} l\right),
$$

where $E_{n}$ is the middle of the $n$th miniband and $t_{n}$ the hopping parameter. Notice that we include the free motion along the $y$ direction. Then, $D(E)$ takes the form

$$
D(E)=\frac{\sqrt{2 m} A}{\pi h} \sum_{n} \int_{-\pi / l}^{\pi / l} \frac{d k_{x}}{\sqrt{E-E_{n}+2 t_{n} \cos \left(k_{x} l\right)}}
$$

and evaluate the integral numerically with $t_{n}$ equal to one quarter of the width of the $n$th miniband (the parameters $E_{n}$ and $t_{n}$ we obtained numerically for the same shape of superlattice as for the relativistic particles).

The results are shown in Fig. 12. The solid red curve is for electrons $\left(D_{0}=4 A / \pi^{2}\right)$, and the dashed blue one for bosons; the left and right scales are different due to the absence of spin degeneracy in the latter case. The dotted green curve is for nonrelativistic electrons in the superlattice in the tight-binding model with $D_{0}=\sqrt{2 m} A / \pi h$ and $m=0.067 m_{0}$. It appears different from the usual 1D one because we included the motion along the $y$ direction but not the one along the $z$ direction. ${ }^{15}$ For comparison, we also show the DOS for the Dirac two-dimensional electron gas (2DEG) in the absence of a superlattice by the red dot-dashed curve. The structure seen in the DOS is directly related to that of the dispersion relation shown in Figs. 5, 6, 8, and 9. The more pronounced 


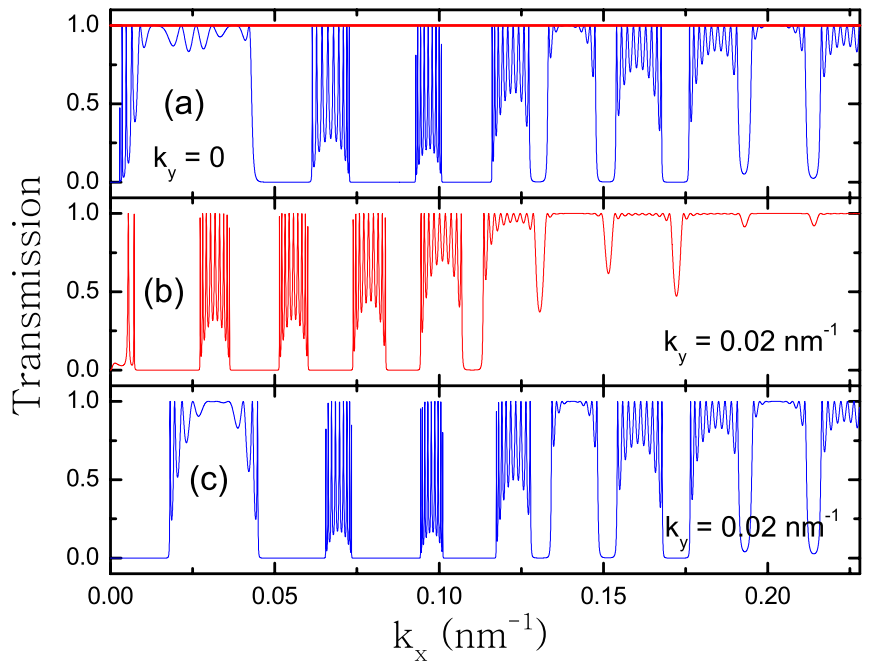

FIG. 11. (Color online) Electrons vs bosons. Plots of the transmission through a structure consisting of ten units with $c$ $=10^{6} \mathrm{~m} / \mathrm{s}, V=50 \mathrm{meV}, a=50 \mathrm{~nm}, b=100 \mathrm{~nm}$, and $m=0$.

structure in the case of bosons reflects the wider gaps, on the average, at the zone boundaries. In general, the DOS increases upon approaching the band edges and then decreases. Accordingly, there are no peaks in the DOS for a Dirac 2DEG, no states with $E<0$ for a nonrelativistic particle, etc.

\section{SUMMARY}

We obtained the dispersion relation for massless fermions and zero-spin bosons using, respectively, the Dirac and Klein-Gordon equations with a 1D periodic potential but allowing for motion of these particles in two dimensions. In doing so, we generalized the treatment of Ref. 12 which considered motion only along one direction. We also evalu-

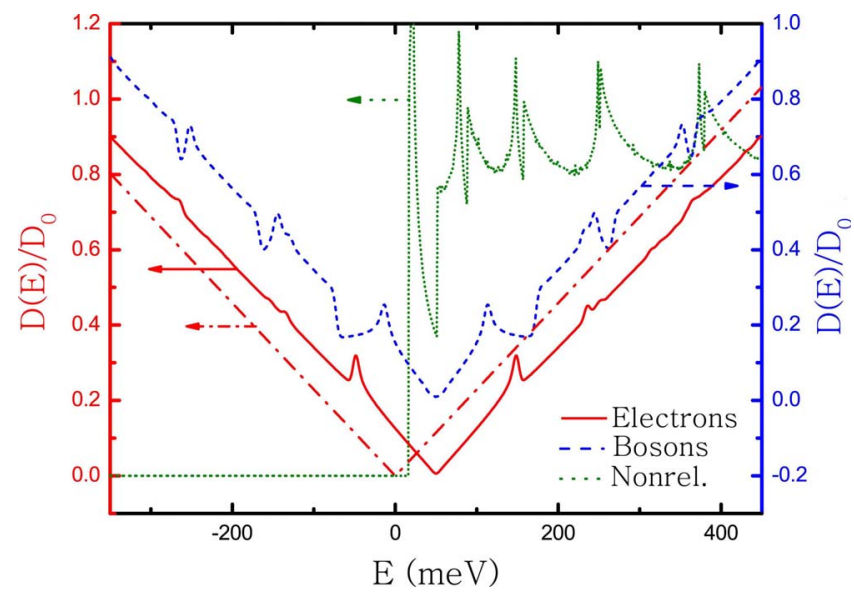

FIG. 12. (Color online) Electrons vs bosons. Density of states $D(E)$ vs energy $E$ for a superlatice with $c=10^{6} \mathrm{~m} / \mathrm{s}, V=100 \mathrm{meV}$, $a=b=10 \mathrm{~nm}$, and $m=0$. The red, solid curve is for electrons, and the blue, dashed one for bosons; the latter is shifted for clarity. The green dotted curve is for a nonrelativistic particle $\left(m=0.067 m_{0}\right)$ in the tight-binding model [see Eq. (38)], and the red dot-dashed curve for the massless Dirac electron in the absence of a superlattice. ated the transmission through such a superlattice and related it to that through a single barrier. Further, we evaluated the density of states for either superlattice, for a nonrelativistic particle, and for the Dirac massless fermion.

The parameters we chose for fermions are appropriate to graphene that is currently studied intensively. It is well established that electrons in graphene behave effectively as chiral, relativistic massless $(m=0)$ particles with a light speed equal to their Fermi velocity. Though the Dirac and Klein-Gordon equations differ significantly, we chose the same parameters for zero-spin bosons for a more meaningful contrast between the results derived from them. The expression for the wave vector $K$ [Eq. (2)] indicates that taking $m$ $\neq 0$ is equivalent to assuming a higher effective $k_{y}$, through $k_{y}^{* 2}=m^{2} c^{2} / \hbar^{2}+k_{y}^{2}$ or a higher effective mass through $m^{* 2}$ $=\hbar^{2} k_{y}^{2} / c^{2}+m^{2}$. Incidentally, as we pointed out after Eq. (33), taking $m \neq 0$ gives a finite group velocity $v_{k_{y}}$ in the KleinGordon case (see Figs. 8 and 9). We notice in passing that despite infinite velocities encountered in problems studied with the Klein-Gordon equation, the equation is quite versatile, as it can also describe waves in plasmas, torsion-coupled pendula, pulse propagation in periodic dielectric structures, as well as tunneling in waveguides of photonic band-gap materials (see Ref. 15 and references cited therein).

As expected, we saw that Klein tunneling, ${ }^{5-7}$ the perfect transmission of carriers, upon normal incidence $\left(k_{y} \neq 0\right)$, occurs not only through a single potential barrier but also through a superlattice (see Figs. 3 and 11). As pointed out, this difference from the nonrelativistic case occurs because the wave vector $K$ does depend on $k_{y}$ in the former case but not in the second. For zero-spin bosons, this perfect transmission occurs for a highly restricted range of wave vectors (cf. Figs. 2, 10, and 11). The difference though in the transmission between fermions and zero-spin bosons is progressively less pronounced if the incidence is oblique $\left(k_{y} \neq 0\right)$ (see Figs. 2-4 and 11). Apart from these differences though, it is essential to emphasize and realize that a linear spectrum is not an imperative requirement in order to have perfect transmission through potential barriers, for normal incidence, i.e., $T=1$, whereas chirality is indeed essential (cf. Figs. 2-4 and 11).

Finally, we saw the various DOSs contrasted in Fig. 12. Notice that in the DOS for a nonrelativistic superlattice, we retained the motion along the $y$ direction [cf. Eq. (38)]. Apart from being meaningful or instructive, the differences between the various cases shown will be reflected in the transport coefficients, postponed to a future study, and hopefully of use to pertinent experiments.

\section{ACKNOWLEDGMENTS}

This work was supported by the Flemish Science Foundation (FWO-V1), the Belgian Science Policy (IAP), the Brazilian council for research $(\mathrm{CNPq})$, and the NSERC Grant No. OGP0121756.

\section{APPENDIX}

Below, we evaluate the transmission for electrons. The solution to Eq. (5) is a linear combination of two indepen- 
dent solutions $\phi_{a}(x)$ and $\phi_{b}(x)$. Outside the barrier, we have

$$
\Omega_{\mathbf{k}_{x}}(\mathbf{x})=\left(\begin{array}{cc}
1 & 1 \\
\lambda_{+} & -\lambda_{-}
\end{array}\right) N_{k_{x}} e^{i k_{x} x \sigma_{z}},
$$

where $N_{k_{x}}$ is a normalization factor, $\lambda_{ \pm}$and $\Lambda_{ \pm}$are defined as

$$
\lambda_{ \pm}=\frac{k_{ \pm} \hbar c}{E^{\prime}+m c^{2}}, \quad \Lambda_{ \pm}=\frac{K_{ \pm} \hbar c}{\left|E^{\prime}\right|+m c^{2}},
$$

and $\boldsymbol{\Omega}_{k_{x}}(x)$ is given by Eq. (15). Inside the barrier,

$$
\begin{aligned}
& \boldsymbol{\Omega}_{K}(x)=\left(\begin{array}{cc}
1 & 1 \\
\Lambda_{+} & -\Lambda_{-}
\end{array}\right) N_{K} e^{i K x \sigma_{z}}, \quad E>V \\
& \boldsymbol{\Omega}_{K}(x)=\left(\begin{array}{cc}
-\Lambda_{-} & \Lambda_{+} \\
1 & 1
\end{array}\right) N_{K} e^{i K x \sigma_{z}}, \quad E<V .
\end{aligned}
$$

We now use the transfer matrix method and express the solutions in terms of $\phi_{a}$ and $\phi_{b}$. Referring to Fig. 1, we have

$$
\begin{gathered}
\psi(x)=\boldsymbol{\Omega}_{k_{x}}(x)\left(\begin{array}{l}
A \\
B
\end{array}\right) \quad \text { in region I, } \\
\psi(x)=\boldsymbol{\Omega}_{K}(x)\left(\begin{array}{l}
C \\
D
\end{array}\right) \quad \text { in region II, } \\
\psi(x)=\boldsymbol{\Omega}_{k_{x}}(x)\left(\begin{array}{c}
F \\
G=0
\end{array}\right) \quad \text { in region III. }
\end{gathered}
$$

The wave function has to be continuous at $x=0$ and $x=W$. This gives

$$
\begin{gathered}
\left(\begin{array}{l}
A \\
B
\end{array}\right)=\boldsymbol{\Omega}_{k_{x}}^{-1}(0) \boldsymbol{\Omega}_{K}(0)\left(\begin{array}{c}
C \\
D
\end{array}\right), \\
\left(\begin{array}{l}
C \\
D
\end{array}\right)=\boldsymbol{\Omega}_{K}^{-1}(W) \boldsymbol{\Omega}_{k_{x}}(W)\left(\begin{array}{c}
F \\
G=0
\end{array}\right),
\end{gathered}
$$

and

$$
\left(\begin{array}{l}
A \\
B
\end{array}\right)=\mathbf{T}\left(\begin{array}{l}
F \\
0
\end{array}\right)
$$

where $\mathbf{T}$ is defined as

$$
\mathbf{T}=\boldsymbol{\Omega}_{k_{x}}^{-1}(0) \boldsymbol{\Omega}_{K}(0) \boldsymbol{\Omega}_{K}^{-1}(W) \boldsymbol{\Omega}_{k_{x}}(W) .
$$

Explicitly, we obtain the following results for the matrix elements $T_{m n}$ of the $2 \times 2$ transfer matrix $\mathbf{T}$. For $E>V$ or $E$ $<0$, the results are

$$
\begin{gathered}
T_{11}=\alpha e^{i k_{x} W}\left[\left|\lambda^{*}+\Lambda\right|^{2} e^{-i K W}-|\lambda-\Lambda|^{2} e^{i K W}\right], \\
T_{12}=2 i \alpha e^{-i k_{x} W}(\lambda-\Lambda)\left(\lambda+\Lambda^{*}\right) \sin (K W), \\
T_{21}=-T_{12}^{*}, \quad T_{22}=T_{11}^{*},
\end{gathered}
$$

where $\alpha=d D / 4 k_{x} K$. For $0<E<V$, the results are

$$
\begin{gathered}
T_{11}=\alpha e^{i k_{x} W}\left[-|\lambda \Lambda-1|^{2} e^{-i K W}+\left|\lambda \Lambda^{*}+1\right|^{2} e^{i K W}\right], \\
T_{12}=2 i \alpha e^{i k_{x} W}\left(-\lambda^{*} \Lambda^{*}+1\right)\left(\lambda^{*} \Lambda+1\right) \sin (K W) \\
T_{21}=-T_{12}^{*}, \quad T_{22}=T_{11}^{*} .
\end{gathered}
$$

With reference to Fig. 1, the current is given by

$$
\begin{gathered}
j_{x}=2 e c N_{k_{x}}^{2} \lambda\left(|A|^{2}-|B|^{2}\right) \quad \text { in region I, } \\
j_{x}=2 e c N_{k_{x}}^{2} \lambda|F|^{2} \quad \text { in region III. }
\end{gathered}
$$

Then, the transmission $\mathcal{T} \equiv \mathcal{T}\left(k_{x}, k_{y}\right)$ is

$$
\mathcal{T}=\frac{|F|^{2}}{|A|^{2}}=\frac{1}{\left|T_{11}\right|^{2}}
$$

Explicitly, for $E>V$, the transmission is given by Eq. (11), whereas for $0<E<V$, it takes the form

$$
\mathcal{T}=\left[4 \alpha^{2} F^{\prime 2} \sin ^{2}(K W)+\cos ^{2}(K W)\right]^{-1},
$$

with $\quad F^{\prime}=\left(K^{2}+k_{y}^{2}\right)\left(k_{x}^{2}+k_{y}^{2}\right) / d^{2} D^{2}+1+2 k_{y}^{2} / d D, \quad d=(E$ $\left.+m c^{2}\right) / \hbar c$, and $D=\left(|E-V|+m c^{2}\right) / \hbar c$. In a similar way, we obtain the transmission for $E<0$, which is the same as that for $E>0$, and the other cases listed in Secs. II and III.

\footnotetext{
*michael.barbier@gmail.com

†rancois.peeters@ua.ac.be

†takis@alcor.concordia.ca

§joaomilton.pereira@ua.ac.be
}

${ }^{1}$ K. S. Novoselov, A. K. Geim, S. V. Morozov, D. Jiang, Y. Zhang, S. V. Dubonos, I. V. Grigorieva, and A. A. Firsov, Science, 306, 666 (2004).

${ }^{2}$ Y. Zhang, Y. W. Tan, H. L. Stormer, and P. Kim, Nature (London) 438, 201 (2005).

${ }^{3}$ Y. Zheng and T. Ando, Phys. Rev. B 65, 245420 (2002).

${ }^{4}$ V. P. Gusynin and S. G. Sharapov, Phys. Rev. Lett. 95, 146801 (2005).

${ }^{5}$ O. Klein, Z. Phys. 53, 157 (1929).
${ }^{6}$ M. I. Katsnelson, K. S. Novoselov, and A. K. Geim, Nat. Phys. 2, 620 (2006).

${ }^{7}$ J. M. Pereira, Jr., V. Mlinar, F. M. Peeters, and P. Vasilopoulos, Phys. Rev. B 74, 045424 (2006).

${ }^{8}$ C. W. J. Beenakker, Rev. Mod. Phys. (to be published).

${ }^{9}$ J. M. Pereira, Jr., P. Vasilopoulos, and F. M. Peeters, Appl. Phys. Lett. 90, 132122 (2007).

${ }^{10}$ J. Schliemann, D. Loss, and R. M. Westervelt, Phys. Rev. Lett. 94, 206801 (2005); W. Zawadzki, Phys. Rev. B 72, 085217 (2005); R. Winkler, U. Zulicke, and J. Bolte, ibid. 75, 205314 (2007).

${ }^{11}$ J. Schwinger, Phys. Rev. 82, 664 (1951); D. Allor, T. D. Cohen, and D. A. McGady, arXiv:0708.1471 (unpublished). 
${ }^{12}$ B. H. J. McKellar and G. J. Stephenson, Jr., Phys. Rev. C 35, 2262 (1987).

${ }^{13}$ W. Greiner, Relativistic Quantum Mechanics (Springer, Berlin, 1998).

${ }^{14}$ A. M. Steinberg, P. G. Kwiat, and R. Y. Chiao, Phys. Rev. Lett.
71, 708 (1993); H. G. Winful, ibid. 90, 023901 (2003); Y. Japha and G. Kurizki, Phys. Rev. A 53, 586 (1996).

${ }^{15}$ G. Bastard, Wave Mechanics Applied to Semiconductor Heterostructure (Les Editions de Physique, Les Ulis, 1988). 